\title{
GaN-Based Materials for Blue Emitting Device Structures Grown in Multiwafer Planetary
}

\section{${ }^{\circledR}$ Reactors}

\author{
O. Schoen ${ }^{1}$, D. Schmitz ${ }^{1}$, M. Heuken ${ }^{1}$, Holger Juergensen ${ }^{1}$ and M. D. Bremser ${ }^{2}$ \\ ${ }^{1}$ AIXTRON AG, \\ ${ }^{2}$ AIXTRON Inc.,
}

(Received Monday, June 22, 1998; accepted Monday, October 19, 1998)

Using optimised growth processes for an AIX 2000 HT Planetary ${ }^{\circledR}$ Reactor a high material quality and high potential device yield are demonstrated. Doping levels for GaN single layers from $1 \cdot 10^{20}$ $\mathrm{cm}^{-3}$ free electrons to semi-insulating to $1 \cdot 10^{18} \mathrm{~cm}^{-3}$ free holes with state-of-the-art layer resistance uniformities especially for n-type layers are shown. Both AlGaN and GaInN with composition homogeneities of better than $1 \mathrm{~nm}$ photoluminescence peak-wavelength standard deviation are displayed. Finally, examination of optically pumped laser action in simple double-hetero structures is quoted to prove the quality of the material.

\section{Introduction}

As device structures of $\mathrm{GaN}$ based materials are produced for commercial application in LEDs and Lasers [1], tools for industrial mass production become necessary. These machines are required to provide material with state-of-the-art characteristics while maintaining high throughput, high reproducibility, high growth efficiency and good uniformity of individual layers for maximal yield of the production line. The AIXTRON multiwafer MOVPE systems with the Planetary ${ }^{\circledR}$ Reactor design are uniquely suited to meet these specifications. The growth processes are continuously optimised regarding total pressure, growth temperature and precursor flows to meet the material quality requirements while the reactor design ensures high yield.

\section{Sample Preparation}

All GaN based structures discussed here were grown with a GaN nucleation layer on sapphire substrates in the (0001) surface orientation. The layers were produced in a AIX 2000 HT Planetary ${ }^{\circledR}$ Reactor where the uniformity of the layer characteristics is ensured by a two fold rotation of the substrates: up to 7 sattelite disks carrying 2" substrates are rotated by the Gas Foil Rotation ${ }^{\circledR}$ principle while the main disk is turned by mechanical drive. The precursors $\mathrm{NH}_{3}, \mathrm{TEGa}, \mathrm{TMGa}$, TMAl, TMIn, $\mathrm{SiH}_{4}$ and $\mathrm{Cp}_{2} \mathrm{Mg}$ are injected in the cen- ter of the main disk together with $\mathrm{N}_{2}$ or $\mathrm{H}_{2}$ as carrier gas, seperated in an upper and a lower flow for MO and hydride precursors respectively. Reactor temperatures up to $1200^{\circ} \mathrm{C}$ and total pressures between 50 and 1000 mbar were used for the growth processes.

\section{Electrical characterization}

Non intentionally doped $\mathrm{GaN}$ layers are semi-insulating or lightly n-type with background electron concentrations below $5 \cdot 10^{16} \mathrm{~cm}^{-3}$. Intentional n-type doping is obtained by introducing $\mathrm{SiH}_{4}$ into the gas phase. Free electron concentrations of up to $1 \cdot 10^{20} \mathrm{~cm}^{-3}$ have been obtained. Figure 1 shows a topology of the sheet resistance of a highly n-doped $0.5 \mu \mathrm{m}$ thick GaN layer mapped by a Lehighton inductive meassurement setup. The average sheet resistance is $15.75 \Omega /$ square with a standard deviation of $0.86 \%$. This indicates excellent doping uniformities taking into account that both thickness distribution and doping homogeneity contribute to this value (see Section 4). Intentional p-type doping has been achieved by using $\mathrm{Cp}_{2} \mathrm{Mg}$ as precursor for the acceptor. With $\mathrm{Mg}$ concentrations of more than $1 \cdot 10^{20} \mathrm{~cm}^{-3}$ hole concentrations up to $1 \cdot 10^{18} \mathrm{~cm}^{-3}$ are obtained. Uniformities for p-type carrier density with a standard deviation around $10 \%$ have been found, revealing incorporation uniformity as well as influences of the activation process. 


\section{Thickness measurement}

The layer thickness of single layer GaN on sapphire structures are routinely examined by white light interference evaluation on a Waterloo PLM 100 wafer mapper system. Figure 2 shows a mapping of a single layer $\mathrm{GaN}$ on sapphire wafer - the average thickness is $2.44 \mu \mathrm{m}$ with a standard deviation of $0.75 \%$. Usual variations for all nitride based materials are in the range of $2 \%$ standard deviation.

\section{Composition homogeneity of ternary compounds}

The Waterloo PLM 100 photoluminescence (PL) mapper was used to study the distribution of the peak wavelength of the bandgap related emission line of ternary layers at room temperature. Structures of GaInN and $\mathrm{AlGaN}$ layers on a $\mathrm{GaN}$ buffer on sapphire have been thus examined. Figure $3 \mathrm{a}$ shows the wafer mapping (left) and wavelength distribution (right) for a GaInN layer with an estimated average of 6 to $8 \% \mathrm{InN}$ in the lattice - the average wavelength is $382.44 \mathrm{~nm}$ with a standard deviation of $0.94 \mathrm{~nm}$. For a AlGaN layer with roughly 8 to $12 \% \operatorname{AlN}(\mathrm{S})$ an average peak wavelength of $340.31 \mathrm{~nm}$ and a standard deviation of $0.26 \mathrm{~nm}$ were found - see Figure $3 \mathrm{~b}$. The standard deviation for emitted intensity is typically less than $10 \%$ for both ternary systems.

\section{Material property qualification by device fabrication}

A simple double-hetero (DH) structure consisting of a $10 \mathrm{~nm} \mathrm{GaN}$ cap, $50 \mathrm{~nm}$ GaInN with ca. $16 \% \mathrm{InN}$ (S) and $1.6 \mu \mathrm{m} \mathrm{GaN}$ buffer was cleaved into roughly $1 \mathrm{~mm}$ wide pieces and exposed to high intensity optical pumping with a pulsed $\mathrm{N}_{2}$ laser. [2] At excitation densities up to $1 \mathrm{MW} / \mathrm{cm}^{2}$ the emission from that sample was found to concentrate into a single lasing mode at $78 \mathrm{~K}$, as shown in Figure 4. This lasing action without a FabryPerot cavity is evidence of the high optical quality and high gain coefficient of the sample.

\section{Conclusion}

Electrical and optical measurements prove our $\mathrm{GaN}$ material and its ternary alloys to be of high quality. Doping and ternary compositions have been shown to have state-of-the-art homogeneity, except for p-type $\mathrm{GaN}$ where the growth process and activation of the acceptors require further optimisation. These characterization results combined with the high yield due to the unique design of the AIXTRON Planetary ${ }^{\circledR}$ Reactors prove these systems to be an optimal tool for GaN based LED and - in future - laser mass production.

\section{ACKNOWLEDGMENTS}

We hereby gratefully acknowledge the work of G. Yablonskii et al., Institute of Physics, Minsk, on the optical excitation experiments quoted above.

\section{REFERENCES}

[1] S. Nakamura, T. Mukai, M. Senoh, Jpn. J. Appl. Phys. 30, L1998 (1991).

[2] E. Woelk, G. Strauch, D. Schmitz, H. Juergensen, "BlueGreen LED Material Based on III-Nitride MOCVD", Proceedings of International Symposium on Blue Laser and Light Emitting Diodes, Chiba Univ., Japan, 1996, 514-517

[3] G. P. Yablonskii, E. V. Lutsenko, I. P. Marko, O. Schoen, M. Heuken, B. Schineller, A. Gutzeit, M. Schwambera, Lim Peng Huei, K. Heime, "Stimulated Emission and Lasing in GaInN/GaN Heterostructures grown by MOVPE", to be presented at Chiba 1998

\section{FIGURES}

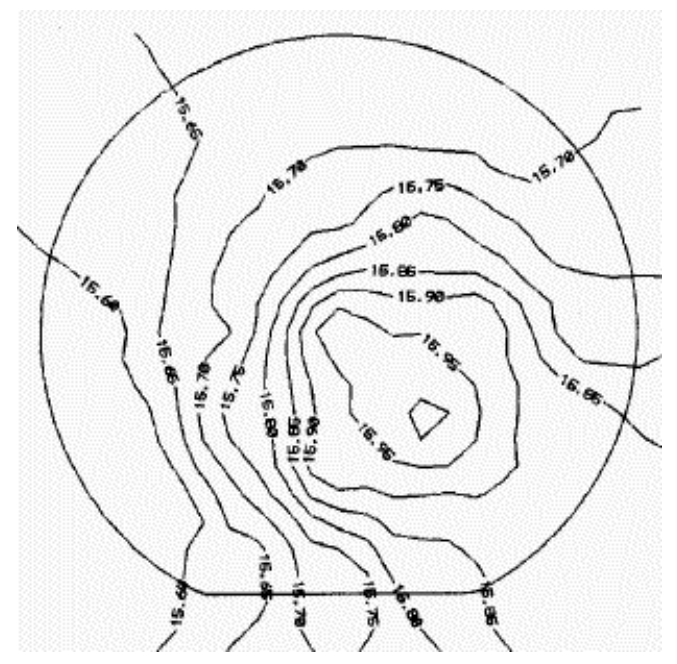

Figure 1. Sheet resistance mapping of a $0.5 \mu \mathrm{m} \mathrm{GaN}$ :Si layer on c-plane sapphire: average resistance is $15.75 \Omega$ /square with a standard deviation of $0.86 \%$

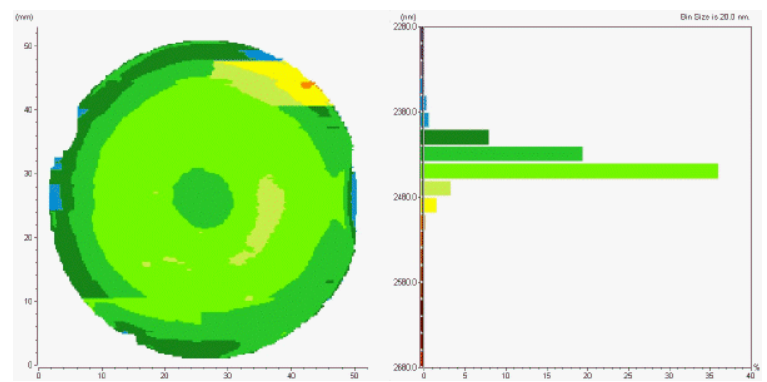

Figure 2. Thickness mapping of a $\mathrm{GaN}$ layer on sapphire substrate: average thickness is $2.44 \mu \mathrm{m}$ with a standard deviation of $0.75 \%$ 

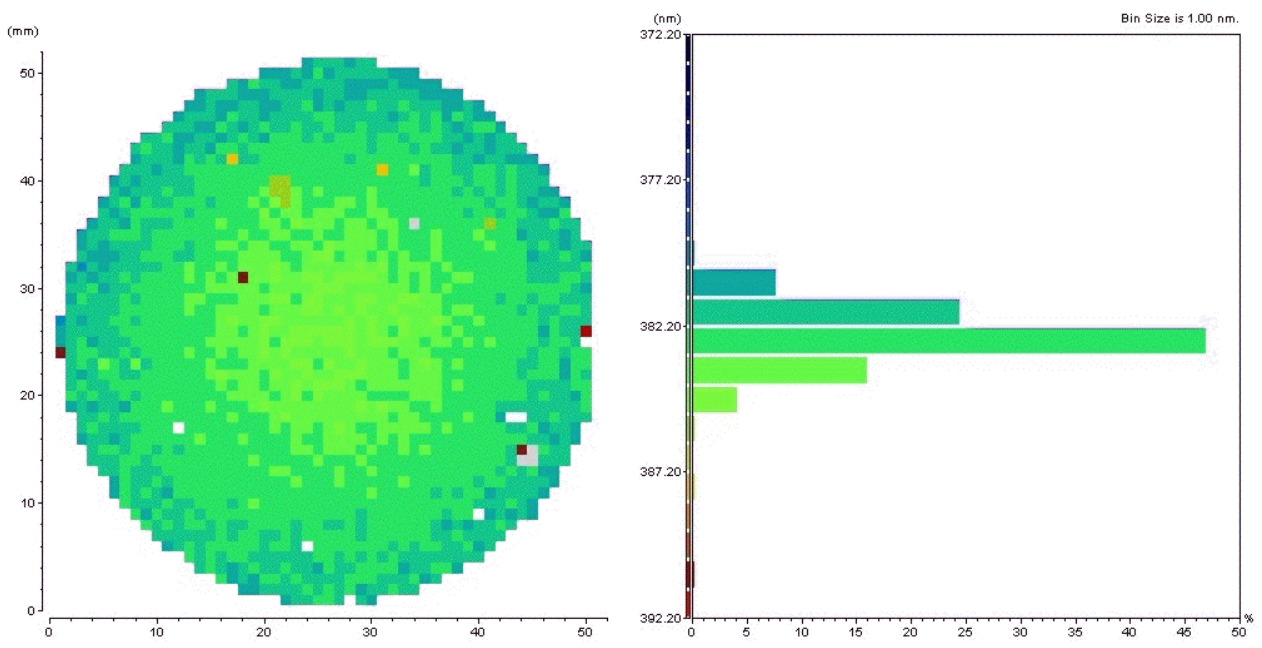

Figure 3a. RT PL peak wavelength mapping of a full 2" wafer GaInN/GaN heterostructure and wavelength distribution: average wavelength is $382.44 \mathrm{~nm}$ with a standard deviation of $0.94 \mathrm{~nm}$ (distribution bin size is $1 \mathrm{~nm}$ )

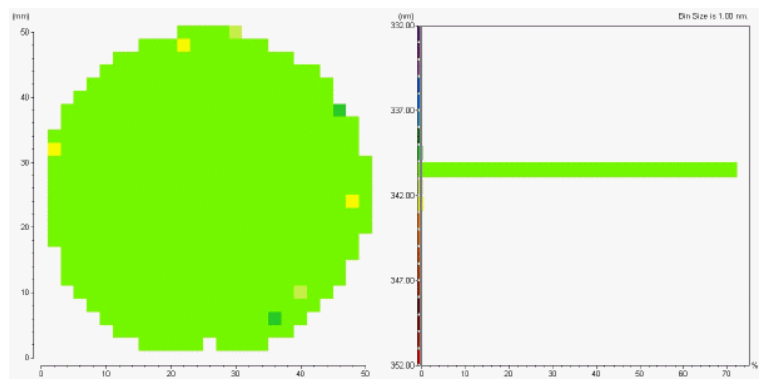

Figure 3b. RT PL peak wavelength mapping of a full 2" wafer AlGaN/GaN heterostructure and wavelength distribution: average wavelength is $340.31 \mathrm{~nm}$ with a standard deviation of $0.26 \mathrm{~nm}$ (distribution bin size is $1 \mathrm{~nm}$ )

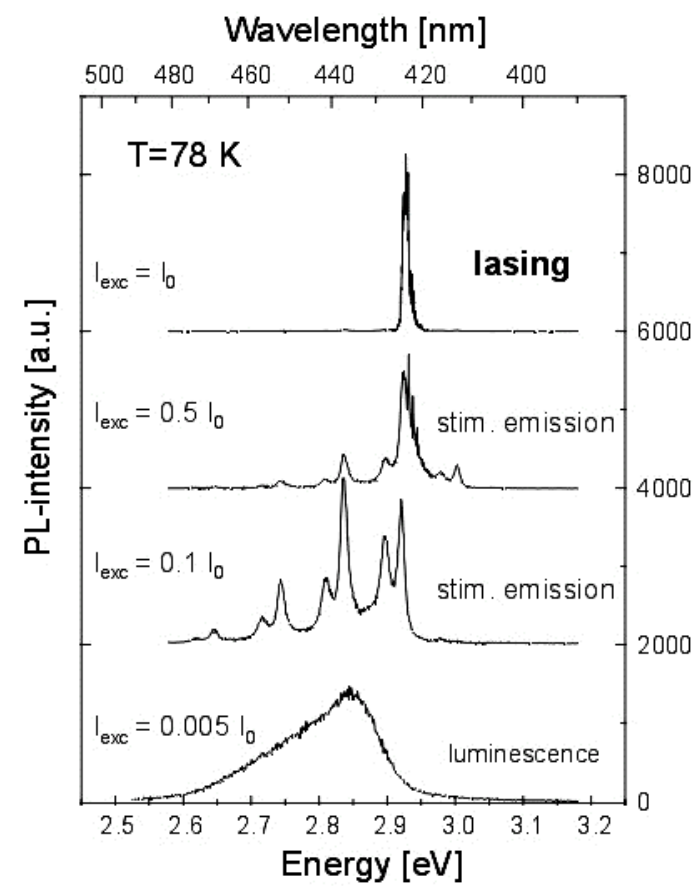

Figure 4. stimulated emission and LT laser action by optical pumping with increasing excitation intensity of a simple GaN/ GaInN DH structure [3] 